\title{
Polymer-Based Cantilevers With Integrated Electrodes
}

\author{
Schahrazede Mouaziz, Giovanni Boero, Radivoje S. Popovic, Member, IEEE, and Jürgen Brugger
}

\begin{abstract}
An innovative release method of polymer cantilevers with embedded integrated metal electrodes is presented. The fabrication is based on the lithographic patterning of the electrode layout on a wafer surface, covered by two layers of SU-8 polymer: a 10- $\mu \mathrm{m}$-thick photo-structured layer for the cantilever, and a $200-\mu \mathrm{m}$-thick layer for the chip body. The releasing method is based on dry etching of a 2 - $\mu$ m-thick sacrificial polysilicon layer. Devices with complex electrode layout embedded in free-standing 500- $\mu \mathrm{m}$-long and 100- $\mu \mathrm{m}$-wide $\mathrm{SU}-8$ cantilever were fabricated and tested. We have optimized major fabrication steps such as the optimization of the SU-8 chip geometry for reduced residual stress and for enhanced underetching, and by defining multiple metal layers [titanium (Ti), aluminum (Al), bismuth (Bi)] for improved adhesion between metallic electrodes and polymer. The process was validated for a miniature $2 \times 2 \mu \mathrm{m}^{2}$ Hall-sensor integrated at the apex of a polymer microcantilever for scanning magnetic field sensing. The cantilever has a spring constant of $\cong 1 \mathrm{~N} / \mathrm{m}$ and a resonance frequency of $\cong 17 \mathrm{kHz}$. Galvanometric characterization of the Hall sensor showed an input/output resistance of $200 \Omega$, a device sensitivity of 0.05 V/AT and a minimum detectable magnetic flux density of $9 \mu \mathrm{T} / \mathrm{Hz}^{1 / 2}$ at frequencies above $1 \mathrm{kHz}$ at room temperature. Quantitative magnetic field measurements of a microcoil were performed. The generic method allows for a stable integration of electrodes into polymers MEMS and it can readily be used for other types of microsensors where conducting metal electrodes are integrated in cantilevers for advanced scanning probe sensing applications.

[1573]
\end{abstract}

Index Terms-Dry etch, Hall sensor, integrated electrodes, polysilicon sacrificial layer, stress-reducing geometries, SU-8 cantilever, thin film metal deposition and lift off.

\section{INTRODUCTION}

$\mathbf{S}$ ENSORS based on cantilevers have seen a rapid growth recently as their relative simple mechanical behavior allows straightforward translation of mechanical forces into displacement. Cantilevers have the additional advantage that micro and nanosensors placed on their distal can be approached to surfaces for investigation of material properties. Microcantilevers were originally made for surface probing in atomic force microscopes (AFM) where typically silicon and silicon nitride is used as cantilever material. Polymer-based devices are interesting alternatives to silicon for selected applications in micro/nano-electro-mechancial-systems (MEMS/NEMS), particularly when the polymer materials can be functionalized for enhanced specific material properties (e.g, optical, electrical, and mechanical). Possible applications include scanning

Manuscript received April 14, 2005; revised September 28, 2005. This work was supported by the CTI/Top Nano 21. Subject Editor J. Judy.

The authors are with the Microsystems Laboratory, Ecole Polytechnique Fédérale de Lausanne (EPFL), 1015 Lausanne, Switzerland (e-mail: schahrazede.mouaziz@epfl.ch).

Digital Object Identifier 10.1109/JMEMS.2006.879376 probe instruments, accelerometers, pressure sensors, but also biomedical microinstruments and microfluidic devices. Many polymer-based materials can be rendered photosensitive which allows the realization of microstructures using lithography and moulding techniques to form quasi-three-dimensional (3-D) devices. For instance, SU-8 [1], [2] is an epoxy-type negative tone photoresist with a low optical absorption in the near UV range spectrum. This allows the micropatterning of thick coatings with vertical sidewalls and high-aspect ratio. Once SU-8 is cross-linked it is chemically stable and resistant to most solvents and acids. It has a very high thermal resistance and good mechanical properties as well. Due to the high-aspect ratio capability it has been used, at the beginning, as mold for electroplating for metallic-microgears [3], microcoils [4], and wheels [5] and as a basic material for fabrication of microfluidic channels [6], [7] and microgears [4], [8]. Later, SU-8 was used also as structural material for micromechanical devices such as AFM cantilevers with integrated moulded nanoscale tips [9]. The fact that SU-8 has about 30 times smaller Young's modulus $E$ than silicon $\left(E_{\mathrm{SU} 8}=4-5 \mathrm{GPa}\right.$ and $\left.E_{\mathrm{Si}}=167 \mathrm{GPa}[10]\right)$ makes SU-8 extremely interesting as cantilever probes. Another interesting concept incorporated SU-8 pillars on the top of silicon cantilevers as additional mass to alter their resonance frequency [11].

Unfortunately, unlike silicon with its semiconducting and piezoresistive properties, $\mathrm{SU}-8$ is a passive material, i.e., it has no inherent properties that can be exploited for sensing applications. To overcome this limitation, two distinct approaches have been proposed recently.

- The first method is to modify the SU-8 itself by incorporating micro and nanoparticles into the polymer. Jiguet et al. added $\mathrm{Ag}$ particles (mean size of the particles is about $1.1 \mu \mathrm{m})$ to make SU-8 conducting [12]. Gammelgaard et al. developed an SU-8/carbon composite by mixing carbon-black particles into SU-8 [13]. In both cases the composite material could still be structured by standard UV lithography although the resolution was deteriorated because of the particle induced scattering of the UV light used to expose the resin.

- The second possibility is to embed electrode structures on the surface of SU-8. This was first shown by Kim et al. where a self-assembled monolayer (SAM) was used as anti-adhesion coating for a dry mechanical release [14]. Thaysen $e t$ al. showed recently a first generation of SU-8 cantilevers with integrated metallic strain gauge for use in AFM or chemomechanical transducers [15].

In this paper, we present a microfabrication method for polymer-based cantilevers with integrated electrodes suitable for the realization of cantilever sensing systems technique. 

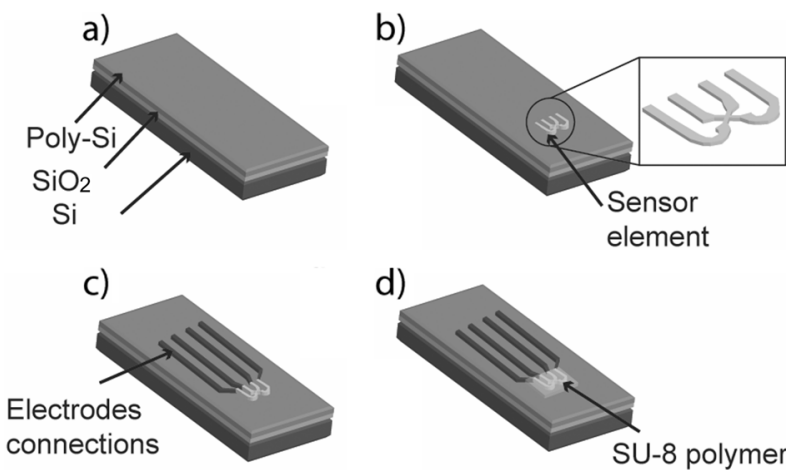

d) connections
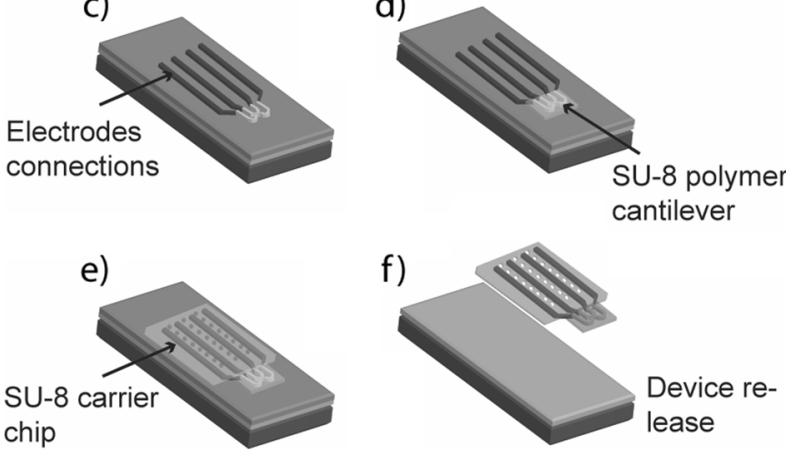

Fig. 1. Schematic illustration of the process for SU-8 cantilever with integrated electrodes. (a) Silicon wafer with $0.5 \mu \mathrm{m}$ of thermal oxide on which $2 \mu \mathrm{m}$ of polysilicon sacrificial layer is deposited. (b) Electrodes (e.g., micro Bi-Hall sensor) realized with lift off. (c) Thin metallic film structured with lift off to pattern the electrodes. (d) and (e) Thin and thick SU-8 photoresist layers spin-coated on the patterned thin metal films and treated with the suitable, low stress process.(f) Release the devices by plasma etching of the polysilicon.

The novelty in this microfabrication technology is the use of polysilicon as a sacrificial layer removed by a dry-etching technique. As demonstrator we present a new micron-scale magnetic field sensor based on a bismuth (Bi) Hall-probe integrated at the distal end of a SU-8 cantilever. The highly sensitive Bi Hall-probe can be scanned across the sample and measure the magnetic stray field with high spatial resolution. In view of our application, $\mathrm{Bi}$ is the appropriated material for the micro-Hall sensors.

However the method is compatible with a variety of other metallic thin films.

\section{EXPERIMENTAL PROCESS}

\section{A. Hall Sensors and Electrodes}

A silicon wafer with $0.5 \mu \mathrm{m}$ of thermally grown silicon dioxide is used as a substrate. A $2-\mu \mathrm{m}$-thick layer of polysilicon is deposited by low-pressure chemical vapor deposition (LPCVD) and used at the end of the process as a sacrificial layer to release the devices [Fig. 1(a)]. Standard negative tone resist (MAN 1410) photolithography is used to pattern with lift off the $\mathrm{Bi}$, the material used to produce Hall sensors [see Fig. 1(b)]. Bi is a semimetal with a carrier concentration fifth-order magnitude smaller than metals, low resistivity, and negligible surface charge depletion effects, the usual limiting factor to the miniaturization of semiconductor devices [16]. Hexamethyldisulazane (HMDS) adhesion promoter is used in an oven at $150{ }^{\circ} \mathrm{C}$ to enhance the bonding of the photoresist to the polysilicon surface. Then, a $1.35-\mu$ m-thick MAN 1410 negative photoresist is spin-coated in Rite Track, an automatic spin coater, at $2000 \mathrm{rpm}$ for $45 \mathrm{~s}$. The resin is baked $90 \mathrm{~s}$ at $98{ }^{\circ} \mathrm{C}$, exposed to the UV light during $40 \mathrm{~s}$ (corresponding time to an energy of $370 \mathrm{~mJ} / \mathrm{cm}^{2}$ ) and developed in a beaker in a time of $90 \mathrm{~s}$. Evaporation of $\mathrm{Bi}$ is carried out by Joule effect in Alcatel EVA 600 evaporator. The thickness and the rate of deposition are $300 \mathrm{~nm}$ and $5 \AA / \mathrm{s}$, respectively. Metal lift off is done first in a bath of EC solvent for $120 \mathrm{~min}$; then, in a second bath of acetone for $60 \mathrm{~min}$. The wafers are cleaned in isopropanol, quick dump rinse (QDR), and ultraclean rinse, consecutively.

The electrodes made with two metallic thin films, Ti-Al are structured by lift off in the same way as Bi. After photolithography of $1.35-\mu \mathrm{m}$ MAN 1410, Ti and $\mathrm{Al}$ are deposited successively by e-beam evaporation with Alcatel EVA 600 [see Fig. 1(c)]. The thickness and rate of deposition are $20 \mathrm{~nm}$ at $5 \AA / \mathrm{s}$ for Ti and $280 \mathrm{~nm}$ at $10 \AA / \mathrm{s}$ for Al. Metals lift off is similar to aforementioned $\mathrm{Bi}$ thin film. The choice of the bilayer Ti-Al is based on the fact that the SU-8 has a good adhesion on the $\mathrm{Al}$ but the direct electrical contact between the $\mathrm{Bi}$ and $\mathrm{Al}$ is not optimal.

\section{B. SU-8 Polymer Cantilevers and Carrier Chip}

After dehydration of the substrate in the oven at $150{ }^{\circ} \mathrm{C}$ for 20 min, an initial thin layer of SU-8 (2007 Microchem) is spincoated in RC8 spin-coater to produce cantilevers of the following dimensions: $500-\mu \mathrm{m}$ length, $100-\mu \mathrm{m}$ width, and $10-\mu \mathrm{m}$ thickness [see Fig. 1(d)]. The spin-coating is done in two ramps: the first at $1000 \mathrm{rpm}$ for $5 \mathrm{~s}$ and the second at $1500 \mathrm{rpm}$ for $40 \mathrm{~s}$. Stress control is achieved by a careful adjustment of all thermal treatments during the process. This step is extremely important to reduce the mechanical stress on the cantilevers and to improve the adhesion of the metallic structures on the SU-8 cantilevers. The softbake and the postexposure bake programs are composed of temperature ramps of $1{ }^{\circ} \mathrm{C} / \mathrm{min}$ with a waiting time of $50 \mathrm{~min}$ at $65^{\circ} \mathrm{C}$ and then $60 \mathrm{~min}$ at $95^{\circ} \mathrm{C}$. This is a relatively longer than usual postbake procedure, but is well optimized to reduce the stress significantly. Because of light reflection from the metal layers below the SU-8 layer, the energy of the UV exposure is reduced to the lowest possible value, which in our case is $100 \mathrm{~mJ} / \mathrm{cm}^{2}$. The photoresist is developed with propyleneglycol monomethyl ether acetate (PGMEA) for 2 min and $30 \mathrm{~s}$, rinsed in isopropanol, and dried carefully in a nitrogen gas stream. An oxygen plasma treatment is necessary to clean the substrate surface, which is carried out using $500 \mathrm{~W}$ of $2.45 \mathrm{GHz}$ RF power for $30 \mathrm{~s}$ in Tepla 300 . This preceded the spin-coating in the RC 8 of the thick SU-8 (50 Microchem) photoresist, a layer to build a carrier chip structure of $2.5-\mathrm{mm}$ width, $4.5-\mathrm{mm}$ length and $200-\mu \mathrm{m}$ thickness [see Fig. 1(e)]. First ramp is at $400 \mathrm{rpm}$ for $5 \mathrm{~s}$ and the second is at $600 \mathrm{rpm}$ for $40 \mathrm{~s}$. This layer is prebaked at $65^{\circ} \mathrm{C}$ for $60 \mathrm{~min}$ and then ramping the temperature at $1{ }^{\circ} \mathrm{C} / \mathrm{min}$ to $95^{\circ} \mathrm{C}$ for $120 \mathrm{~min}$. The layer is exposed with an energy of $600 \mathrm{~mJ} / \mathrm{cm}^{2}$, and post baked in the same way as it is prebaked. Finally, the SU-8 layer is developed in PGMEA during $23 \mathrm{~min}$ and rinsed with isopropanol.

\section{Devices Release}

A crucial step is to detach the fragile elements from the surface via a sacrificial layer technique without altering their performance. In the past, several releasing methods using wet chemistry have been presented for SU-8 based devices, such as positive tone photoresist or Ti etching by hydrofluoric (HF) 
acid [5] and by enhanced sacrificial layer etching [17]. It has also been shown that a mechanical dry-release technique based on the anti-adhesion coating of a self-assembled monolayer (SAM) film as sacrificial layer can be used [14]. The advantage of using SAM is that surface features down to the nanometer scale can be replicated into the polymer film due to the extremely thin SAM layer $(<2 \mathrm{~nm})$. This enabled the realization of metal-coated photoplastic probes for scanning near-field optical applications (SNOM) with sub-70-nm optical resolution. The disadvantage of the SAM release technique is that the relatively high force needed to detach the photoplastic elements from the surface can only be applied to solid and compact devices, and not to mechanically compliant elements such as elastic flexible cantilevers. Recently, Zou et al. presented scanning probe microscopy (SPM) probes made with a metal tip and a polymer handle. The wet etching release was based on a copper sacrificial layer removed by a mixture of acetic acid, hydrogen peroxide, and water [18]. Metz et al. showed the technique of anodic dissolution of sacrificial metal layers for the complete or partial detachment of microstructures [19].

In our case, the release of the whole device containing metals integrated to the cantilevers and the carrier chip is performed in an Alcatel 601, a high inductively coupled plasma (ICP) reactor using $\mathrm{SF}_{6}$ at $20^{\circ} \mathrm{C}$ to dry etch the polysilicon sacrificial layer [see Fig. 1(f)]. Due to its high selectivity compared to the polysilicon $(>200)$, the $\mathrm{SiO}_{2}$ is used as an etching stop layer. The devices are released in about 15 min with a rate of under dry etch of about $3.5 \mu \mathrm{m} / \mathrm{min}$. The dry-etching time depends strongly on the mask design shape, which is discussed in detail in Section III-A.

\section{RESULTS AND DISCUSSIONS}

\section{A. Optimized Structural Geometry for Improved Underetching and Stress Release in SU-8 Structure}

In order to easily handle the probes, a bulk chip of millimeter size $\left(4.5 \times 2.5 \mathrm{~mm}^{2}\right.$ in our case $)$ is necessary. In addition, the presence of four electrodes that establish the contact to the electronic part of the setup requires a mechanically stable bulk chip. The release by dry etching of a chip with dimensions in the millimeter range requires more than $60 \mathrm{~min}$ when using the aforementioned etching parameters. This has a negative impact on microstructures released after a short time (e.g., Bi is etched with the fluorine reactive etching gas [20]). We investigated new mask designs to find the shape that leads to the shortest under dry-etch time in order to protect the delicate materials ( $\mathrm{Bi}$ in our case) from a long exposition etching time, a compromise between the stability of the bulk chip and the under etch time. Two decisive points for the mask designs are taken in consideration. First, any large structure needs to contain holes which enable the reactive gases to penetrate and etch the sacrificial layer. Second, the size of the holes has to be as large as possible. Different designs are conceived that help to determine the most efficient structures. In a carrier chip having diamond-shape, features of 270- $\mu \mathrm{m}$ size (see Fig. 2) are underetched, while in honeycomb pattern (see Fig. 3), the structures to release have only

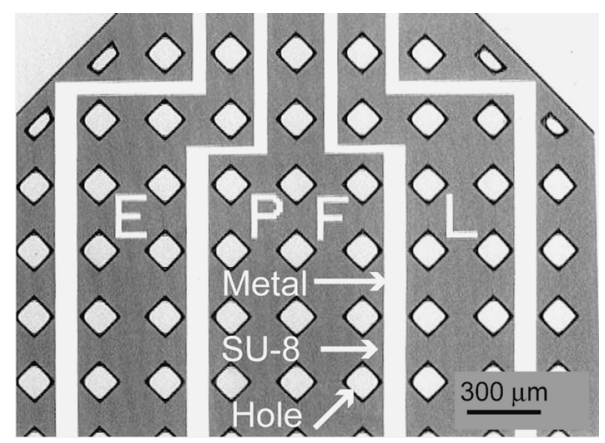

Fig. 2. Optical image of SU-8 chip with diamond shape holes and four embedded metal electrodes. For this design, the time for dry etch of the polysilicon sacrificial layer is $80 \mathrm{~min}$ (which is too long for the exposed metal).

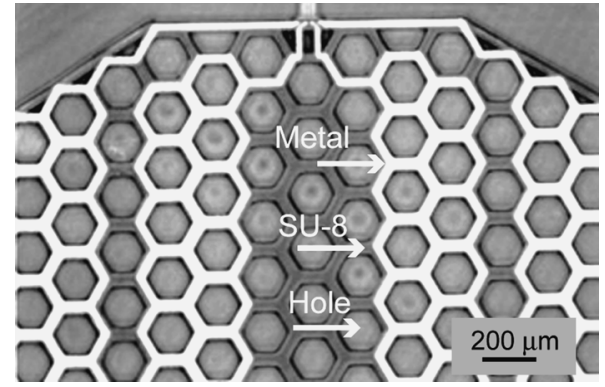

Fig. 3. Optical image of SU-8 structure with embedded metal electrodes. The time for dry etch of this geometry is $15 \mathrm{~min}$ (which is an acceptable time for the exposed metal).

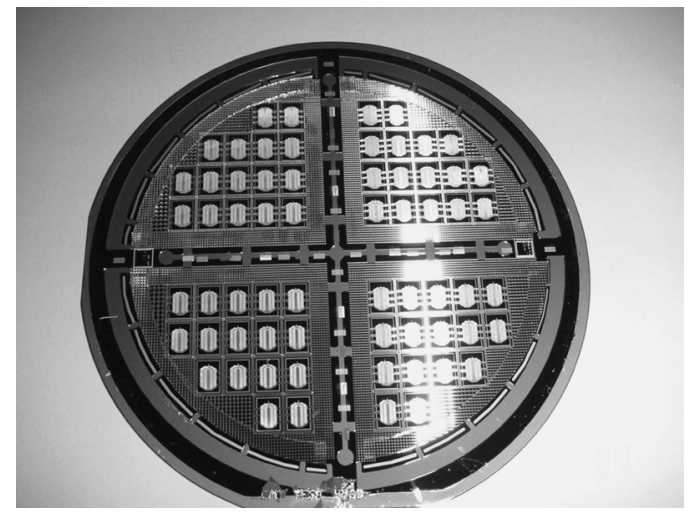

Fig. 4. Photograph of a full wafer $(100 \mathrm{~mm})$ that contains 64 chips after the dry-etch release. The devices stay fixed to the wafers by means of six bars that are broken before using the device.

$50-\mu \mathrm{m}$ size. The time to release the carrier chip with small diamond-shaped holes is almost six times longer than for the honeycomb pattern.

\section{B. Fabricated Devices}

An optical image of a 100-mm wafer after the finished process is shown in Fig. 4. The wafer contains 68 cantilever chip devices that are still attached to a rim on the wafer by six tiny SU-8 bars. The devices are handled individually by detaching them from the wafer after breaking off the bars using tweezers. Fig. 5 shows the Al-Ti metallic tracks on the SU-8 carrier chip demonstrating that the metal film adheres very well to the SU-8. This allows electrical connection by 


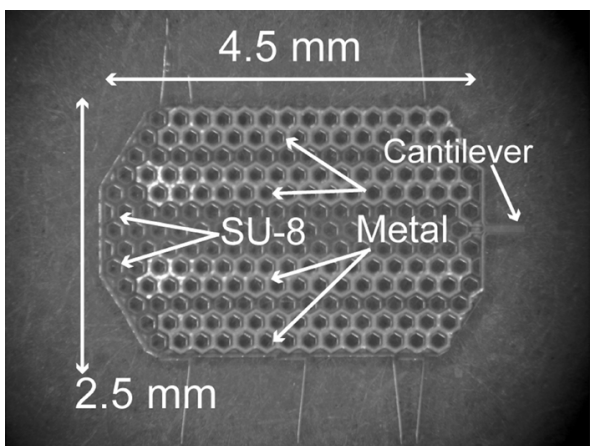

Fig. 5. Photograph of a complete device made with SU-8 thick photoresist for the carrier chip and on the top four metallic electrodes of Ti-Al.

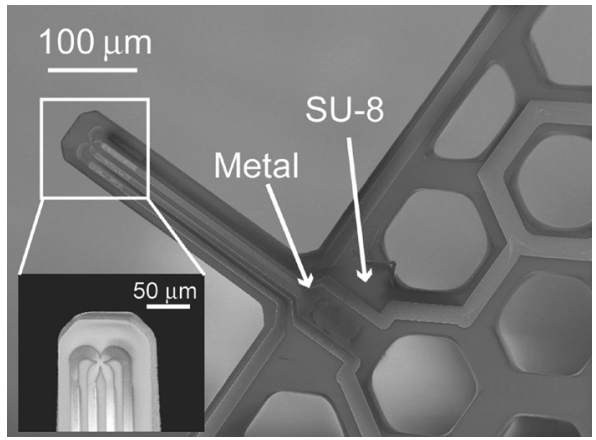

Fig. 6. Scanning electron microscopy image after dry-etch release of the device. The patterned Ti-Al bilayer has a good adhesion to the SU-8 cantilever and carrier chip. A Hall sensor of $2 \times 2 \mu \mathrm{m}^{2}$ active area made with $300-\mathrm{nm}$ thickness Bi thin film is integrated at the edge of the SU-8 cantilever.

means of standard miniaturized metallic microprobe needles or other chip-to-chip interconnects using spring-like clamps. The patterned $\mathrm{Bi}$ on the cantilever shows excellent adhesion as well; no delamination has been observed. A complete Hall sensor on SU-8 cantilever is shown in Fig. 6.

\section{Mechanical Characteristics of the Cantilevers}

The SU-8 cantilever with the metallic tracks has been mechanically characterized with an interferometer and the results have been confirmed by laser beam deflection in an AFM setup. The SU-8 and the multiple layers of metal (Al-Ti-Bi) are sufficient to reflect the laser light. For a rectangular cantilever of the 500- $\mu \mathrm{m}$ length, $100-\mu \mathrm{m}$ width, and $10-\mu \mathrm{m}$ thickness, the resonance frequency is $\cong 17 \mathrm{kHz}$ and the estimated spring constant is $\sim 1 \mathrm{~N} / \mathrm{m}$. The measured quality factor in air is about 30 . Long-term measurements over several days of continuous operation with a vibration amplitude of $200 \mathrm{~nm}$ rms have shown no noticeable deterioration of the mechanical and electrical characteristics of the sensor performance and resonance frequency. It demonstrates the stable integration of metal electrodes in the SU-8 cantilever device.

\section{Galvanomagnetic Characteristics of Hall Sensor}

The Bi Hall sensor shown in Fig. 6 has been electrically and magnetically characterized. The input/output resistance of the sensor is $200 \Omega$. The maximum current applied is $4 \mathrm{~mA}$. Up to this current value the voltage measured stays constant and then fluctuations appears due to the increase of the resistance.

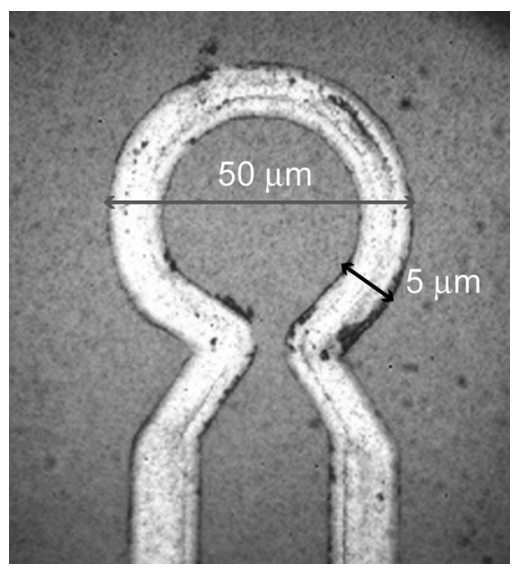

Fig. 7. Optical image of microcoil used to calibrate the Hall sensors. The diameter of the microcoil is $50 \mu \mathrm{m}$ and the width is $5 \mu \mathrm{m}$.

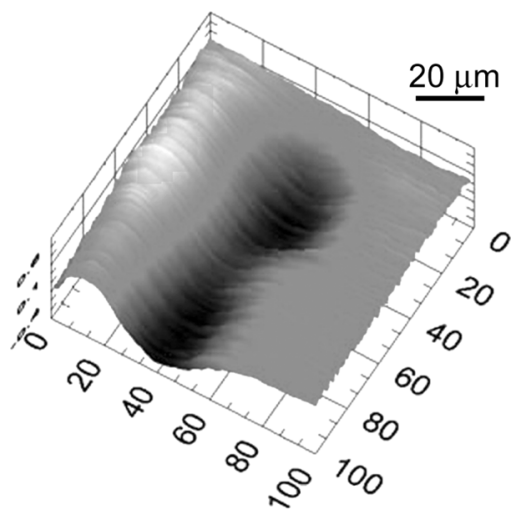

Fig. 8. Magnetic field image of the microcoil shown in Fig. 7 measured with the Hall sensor represented in Fig. 6.

The sensor sensitivity is $0.05 \mathrm{~V} / \mathrm{AT}$ whereas the minimum magnetic flux density detectable is $9 \mu \mathrm{T} / \mathrm{Hz}^{1 / 2}$ above $1 \mathrm{kHz}$ at room temperature. A preliminary magnetic imaging experiment has been carried out over a microcoil of $50 \mu \mathrm{m}$ diameter made with $\mathrm{Al}(300 \mathrm{~nm})$ deposited on $\mathrm{SiO}_{2}$ on a $\mathrm{Si}$ substrate as shown in Fig. 7. With a coil current of $20 \mathrm{~mA}$, the magnetic field at the coil center is about $500 \mu \mathrm{T}$. A scanning Hall probe microscope image of the microcoil (shown in Fig. 8) proves the feasibility of magnetic imaging with Bi Hall probes integrated on SU-8 cantilever. The scanning range $\left(100 \times 100 \mu \mathrm{m}^{2}\right)$ is in $\mathrm{XY}$ plan and the separating distance ( $1 \mu \mathrm{m}$ in our case) between the sample and the sensor is in Z-axis, the direction of the detected magnetic field coming from the microcoil.

\section{CONCLUSION}

Our experiments show that a dry-etching technique of a sacrificial polysilicon layer is feasible and reproducible to release polymer cantilever devices, formed with integrated electrodes on SU-8 support. The releasing technique is clean, fast, and reliable, and can be used for many applications as long as the materials involved are compatible with the ICP etch gases. The releasing time depends on the mask design that has to be adapted to the shapes of the devices and the materials used in the process. We have proven that the cross-linked SU-8 is a suitable material to integrate patterned metals as it shows a high resistance 
to the etch gas $\mathrm{SF}_{6}$. The thermal treatment of SU-8 has been optimized in order to reduce the mechanical stress and improve the adhesion of the electrodes. There is no apparent mechanical stress on the SU-8 structures nor damage in general to the SU-8 cantilevers or on the carrier chip, even after a long etch times. The method has been developed and validated for the fabrication of a micro-Hall sensor on cantilever for scanning magnetic microscopy. A microstructured Bi cross is connected with four conducting wires $\mathrm{Ti} / \mathrm{Al}$ to contact pads leading to the external readout electronics.

Due to its interesting chemical, mechanical, and optical properties, SU-8 is a very promising material for MEMS/NEMS applications. The generic technology described in this paper can be considered as a window for new opportunities. It can readily be applied to the fabrication of microcoils, similar to those shown in Fig. 7, for electron spin resonance microscopy and integrated four-point microprobes for electrical characterization of metallic thin films, etc., [21]. The described technology opens the way to further advances in the field of functional polymer sensors combined with surface microfabrication techniques. The devices are constructed layer by layer (metal and photoplastic polymer) on a planar surface offering all advantages of microfabrication methods, including low-cost massfabrication potential.

\section{ACKNOWLEDGMENT}

The authors would like to thank the Microsystems Laboratory, Ecole Polytechnique Fédérale de Lausanne (EPFL) colleagues for stimulating discussion and the CMI-EPFL cleanroom staff for the very professional process consulting.

\section{REFERENCES}

[1] N. C. LaBianca, J. D. Gelorme, K. Y. Lee, E. Cooper, E. O'Sullivan, and J. M. Shaw, "High aspect ratio optical resist chemistry for MEMs applications," in Electrochem. Soc. Proc., 1995, pp. 386-396.

[2] M. Despont, H. Lorentz, N. Fahrni, J. Brugger, P. Renaud, and P. Vettiger, "Hight-aspect-ratio, ultratick, negative-tone near-UV photoresist for MEMS applications," in MEMS 97 Proc.-IEEE 10th Annu. Int. Workshop Micro Electro Mechanical Systems-An Investigation of Micro Structures, Sensors, Machines and Robots, 1997, pp. 518-522.

[3] H. Lorentz, M. Despont, N. Fahrni, J. Brugger, P. Vettiger, and P. Renaud, "High-aspect-ratio ultrathick, negative-tone near-UV photoresist and its applications for MEMS," Sens. Actuators A, Phys., vol. 64, pp. 33-39, 1998.

[4] H. Lorentz, M. Despont, N. Fahrni, and P. Renaud, "SU-8: a low cost negative resist for MEMS," J. Micromech. Microeng., vol. 7, pp. 121-124, 1997.

[5] L. Dellmann, R. Roth, R. Beuret, G. A. Racine, H. Lorentz, M. Despont, P. Renaud, P. Vettiger, and N. F. de Rooij, "Fabrication process of high aspect ratio elastic and SU-8 structures for piezoelectric motor applications," Sens. Actuators A, Phys., vol. 70, pp. 42-47, 1998.

[6] L. J. Guerin, M. Bossel, M. Demierre, S. Calmes, and P. Renaud, "Simple and low cost fabrication of embedded micro-channels by using a new thick-film photoplastic," in Proc. Transducers'97, 1997, pp. 1419-1421.

[7] R. J. Jackman, R. Ghodssi, M. A. Schmidt, and K. F. Jensen, "Microfluidic systems with on-line UV detection fabricated in photodefinable epoxy,” J. Micromech. Microeng., vol. 11, pp. 263-269, 2001.

[8] A. Bertsch, H. Lorentz, and P. Renaud, "3D micofabrication by combining microstereolithography and thick resist UV lithography," Sens. Actuators A, Phys., vol. 13, pp. 14-23, 1999.

[9] G. Genolet, J. Brugger, M. Despont, U. Drechsler, N. F. de Rooij, D. Anselmetti, and P. Vettiger, "Soft, entirely photoplastic probes for scanning force microscopy," Rev. Sci. Instrum., vol. 70, p. 2398, 1999.
[10] M. Hopcroft, T. Kramer, G. Kim, K. Takashima, Y. Higo, D. Moore, and J. Brugger, "Micromechanical testing of SU-8 cantilevers," Fatigue Fracture Eng. Mater. Struct., vol. 28, no. 8, pp. 735-742, 2004.

[11] E. H. Condradie and D. Moore, "SU-8 thick photoresist processing as a functional material for MEMS applications," J. Micromech. Microeng., vol. 12, pp. 368-374, 2002.

[12] S. Jiguet, A. Bertsch, H. Hofmann, and P. Renaud, "SU8-silver photosensitive nanocomposite," Adv. Eng. Mater., vol. 6, no. 9, pp. 719-724, 2004.

[13] L. Gammelgaard, P. Rasmussen, M. Calleja, and A. Boisen, "Polymeric mechanical sensor with integrated strain gauge read-out based on a piezoresistive carbon-black polymer composite material," in Proc. Eurosensors, 2004, pp. 243-244.

[14] G. Kim, B. Kim, M. Liebau, J. Huskens, D. N. Reinhoudt, and J. Brugger, "Surface modification with self-assembled monolayers for nanoscale replication of photoplastic MEMS," J. Microelectromech. Syst., vol. 11, no. 3, pp. 175-181, Jun. 2002.

[15] J. Thaysen, A. D. Yalçinkaya, P. Vettiger, and A. Menon, "Polymerbased stress sensor with integrated readout," J. Phys. D: Appl. Phys., vol. 35 , pp. 2698-2703, 2002.

[16] A. Sandhu, H. Masuda, A. Oral, and S. J. Bending, "Direct magnetic imaging of ferromagnetic domain structures by room temperature scanning Hall probe microscopy using bismuth micro-Hall sensors probe," Jpn. J. Appl. Phys, vol. 40, pp. 524-527, 2001.

[17] G. Genolet, New photoplastic fabrication techniques and devices based on high aspect ratio photoresist Lausanne, Switzerland, Microtechnique Dept., EPFL, 2001, p. 137.

[18] J. Zou, X. Wang, D. Bullen, K. Ryu, C. Liu, and C. A. Mirkin, "A mould-and-transfer technology for fabricating scanning probe microscopy probes," J. Micromech. Microeng., vol. 14, pp. 204-211, 2004.

[19] S. Metz, A. Bertsch, and P. Renaud, "Partial release and detachment of microfabricated metal and polymer structures by anodic metal dissolution," J. Microelectromech. Syst., vol. 14, no. 2, pp. 383-391, Apr. 2005.

[20] M. Köhler, Etching in Microsystem Technology. New York: Wiley, 1999 , p. 207.

[21] S. Keller, S. Mouaziz, G. Boero, and J. Brugger, "Microscopic fourpoint probe based on SU-8 cantilevers," Rev. Sci. Instruments, vol. 76, no. 12, p. 125102, 2005.

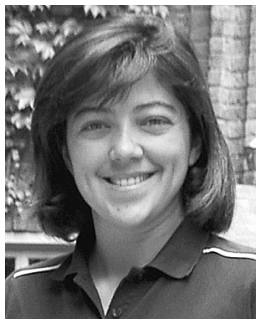

Schahrazede Mouaziz was born in Oran, Algeria, in 1973. She received the Diploma in advanced studies in physics from the University of Es-Senia, Oran, Algeria, in 1995, and the M.S. degree in physics from the University of Neuchâtel, Neuchâtel, Switzerland, in 2001. Currently, she is working towards the Ph.D. degree at the Microsystems and Nanoengineering Laboratory, Ecole Polytechnique Fédérale de Lausanne (EPFL), Lausanne, Switzerland, and the topic of her research is the development of micronano tools for the magnetic microscopy.

She worked as a Research Assistant at the Institute of Applied Optics of the Swiss Federal Institute of Technology, EPFL, Lausanne, Switzerland, in collaboration with the Sensors, Actuators and Microsystems Laboratory of the University of Neuchâtel and NanoWorld Innovative Technologies on the SU-8 AFM cantilevers and hybrid nitride probes.

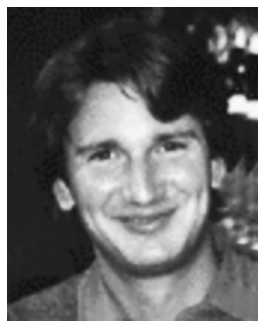

Giovanni Boero was born in Italy, in 1969 . He received the Diploma in physics from the Genoa University, Genoa, Italy, in 1994, and the Ph.D. degree from Ecole Polytechnique Fédérale de Lausanne (EPFL), Lausanne, Switzerland, in 2000. He finished his studies with a Laurea thesis about clusterized gas beams for particle physics experiments, carried out at Fermi National Accelerator Laboratory (FNAL), Batavia, IL

From 1994 to 1996, he was at CERN, Geneva, Switzerland, and at FNAL working mainly on clusterized and atomic gas beams. In 1996, he joined Ecole Polytechnique Fédérale de Lausanne (EPFL), Lausanne, Switzerland, to work on miniaturized nuclear magnetic resonance (NMR) and electron spin resonance (ESR) probes. His current research interests are in the development of innovative devices and methods for magnetic resonance experiments on microscopic samples. 


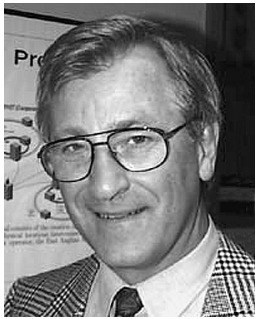

Radivoje S. Popovic (M'82) was born in Yugoslavia (Serbia) in 1945. He received the Dipl.Ing. degree in applied physics from the University of Beograd, Beograd, Yugoslavia, in 1969, and the M.Sc. and Dr.Sc. degrees in electronics from the University of Nis, Nis, Yugoslavia, in 1974 and 1978, respectively.

From 1969 to 1981, he was with Elektronska Industrija Corporation, Nis, Yugoslavia, where he worked on research and development of semiconductor devices and later became head of the company's CMOS department. From 1982 to 1993 , he was with Central R\&D, Landis and Gyr Corporation, Zug, Switzerland, working in the field of semiconductor sensors, interface electronics, and microsystems, where he was responsible for research in semiconductor device physics from 1983 to 1985 , for microtechnology R\&D from 1986 to 1990 , and was appointed Vice President of Central R\&D, in 1991. In 1994, he joined the Swiss Federal Institute of Technology, Ecole Polytechnique Fédérale de Lausanne (EPFL), Lausanne, Switzerland as a Professor of Microtechnology Systems. His current research interests include sensors for magnetic, optical, and mechanical signals, sensor microsystems, physics of submicron devices and noise phenomena.

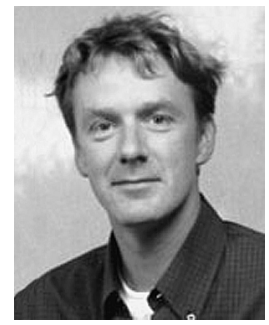

Jürgen Brugger received the Ph.D. degree in 1995 from the University of Neuchâtel, Neuchâtel, Switzerland, for a work on microfabricated tools for the atomic force microscope, which included a one-year period at the Hitachi Central Research Laboratories, Tokyo, Japan.

He then joined the IBM Zurich Research Laboratory, Rüschlikon, Switzerland. From 1998 to 2001, he was directing the "NanoLink" Strategic Research Orientations at the MESA+ Research Institute, University of Twente, Enschede, The Netherlands. In September 2001, he was appointed Assistant Professor "tenure track" at the Microsystems and Nanoengineering Laboratory, Ecole Polytechnique Fédérale de Lausanne (EPFL), Lausanne, Switzerland. He is coinventor of eight patents. His main professional interests include the development of new tools for nanoscience and engineering techniques at the mesoscopic length-scale, in particular to develop methods for accessing the nanometer scale (top-down), and to combine them with self-assembly strategies (bottom-up), and to bridge life-science with solid-state devices at the submicrometer scale.

Dr. Brugger is the elected management board member of the EU Network of Excellence for Nanoelectronics "Phantoms," Steering Board Member of the EU Sixth Framework IP "NaPa," member of the EU NoE "Nano-2-Life," and the Associated Editor of the Journal of Nanoscience and Nanotechnology. He received two IBM research awards. 